$\checkmark$ Research Square

\title{
The Correlation Between the Length of Stay in Post- Acute Care(PAC) and General and Improvements in Stroke Patients
}

Yu-Ju Tung ( $\nabla$ roofyyuju@gmail.com )

Chi Mei Medical Center https://orcid.org/0000-0002-4381-2506

Hsin-Han Cheng

Chi Mei Medical Center

Willy Chou

Chi Mei Medical Center

\section{Research article}

Keywords: Stroke; Subacute care; Rehabilitation; Length of stay; Upper Extremity

Posted Date: April 4th, 2019

DOl: https://doi.org/10.21203/rs.2.1813/v1

License: (c) (i) This work is licensed under a Creative Commons Attribution 4.0 International License.

Read Full License 


\section{Abstract}

Background Post-acute care (PAC) is a transitional care following acute medical stage for stroke patients and offering a more intensive rehabilitative program. According to National Health Insurance in Taiwan, only patients encountered acute stroke within one month, under relative stable medical condition and had potential for aggressive rehabilitation could transfer to PAC institution. Stroke patients receive physical, occupational and speech therapy in PAC. However, there's no research evaluating the effects of PAC for stroke patients ever since the PAC plan inaugurated in Taiwan. Thus, this study aims to investigate whether the duration of hospitalization in PAC correlates to the patients' improvements. Methods This is a retrospective and single- center study in Taiwan. We collected 193 stroke patients who received acute care at Chi Mei Medical Center, Taiwan during 2014 2017 and recorded their length of stay in PAC. Stroke patients' functional ability, such as activities of daily living (ADL) function, swallowing ability and so on, as well as their corresponding scales were assessed on the first and last day during PAC hospitalization. Statistical analysis was conducted via SPSS ver21.0. This study was listed on ClinicalTrials.gov(Identification number: NCT03778905). Results The average duration of hospitalization in PAC was $35.01 \pm 16.373$ days. The longer duration of hospitalization in PAC positively correlates to $\mathrm{BI}$ $(p=0.000 * \star \star, R=0.330), B A L(p=0.000 * \star \star, R=0.461)$, Gait $\operatorname{Speed}\left(p=0.002^{\star \star}, R=0.218\right)$, upper sensory function of $F M A(p=0.000 * \star \star, R=0.263)$ and upper motor function of $F M A(p=0.000 * \star \star, R=0.276)$ in stroke patients significantly. Conclusion Longer duration of hospitalization in PAC is conducive to enhancing ADL function, advancing balance and coordination, boosting walking speed and augmenting both dexterity and sensory function of upper limb in stroke patients.

\section{Introduction}

Post-acute care (PAC) is a transitional care following acute medical stage for stroke patients and offering a more intensive rehabilitative program. According to National Health Insurance in Taiwan, only patients encountered acute stroke within one month, under relative stable medical condition and had potential for better recovery could transfer to PAC institutions. Stroke patients receive physical, occupational and speech therapy in PAC. However, there's no research evaluating the effects of PAC inpatient stays for stroke sufferers ever since the PAC project inaugurated in Taiwan. Thus, this study aims to investigate: Whether the duration of hospitalization in PAC correlates to the stroke patients' improvements?

\section{Methods}

Design

This is a retrospective and single- center study in Taiwan. We collected 305 patients with stroke who received acute care at Chi-Mei Medical Center, Taiwan during 2014 2017 and recorded their length of stays in PAC after the patient discharged from our hospital. Patients' data and performance were collected by PAC case manager from PAC institutions. The study design was approved by the Institutional 
Review Board of Chi Mei Medical Center and was listed on ClinicalTrials.gov(Identification number: NCT03778905).

Study Sample and Enrollment

Inclusion criteria of stroke Post-Acute Care(PAC) are modified Rankin scale(MRS) ranging 2 4, acute onset of stroke within 30 days and over 18-year-old. 305 stroke patients eligible for PAC admission were recruited. 3 stroke patients refused PAC admission while 6 stroke patients had second stroke during acute phase. 296 stroke patients successfully transferred to PAC institution but 8 patients ended PAC hospitalization earlier due to stroke or non-stroke disease progression. We scrutinized the rest of 288 stroke patients and excluded 95 stroke patients with incomplete records of functional ability during PAC hospitalization. 193 stroke patients, with completion of PAC hospitalization and intact data of functional ability, were enrolled in our study (Figure 1).

Functional Ability and Variables

Functional domains of stroke patients, including activities of daily living (ADL) function, swallowing ability, nutritional status, life quality, instrumental ADL function, balance and coordination, walking speed, cardiopulmonary capability, sensory function of upper extremities, motor function of upper extremities, cognitive function, occupational mobility ${ }^{1}$ and language, were scrutinized at the beginning and the end of admission in PAC by the patients' physiatrists and therapists.

Measurements

ADL function was estimated through modified Rankin scale(MRS) and Barthel index(BI), swallowing ability by functional oral intake scale(FOIS), nutritional status via mini nutritional assessment(MNA), life quality through EuroQoL-5D(QoL), instrumental ADL function by Lawton-Brody IADL Scale(IADL), balance/coordination via Berg Balance Test(BAL), walking speed through Usual Gait Speed(Gait Speed), cardiopulmonary capability by Six-Minute Walk Test(6MWT), sensory and motor function of upper extremities via Fugl-Meyer Assessment(FMA), cognitive function through mini-mental state examination (MMSE), occupational mobility/occupational performance by Motor Activity Log(MAL) of amount use and quality and language via concise Chinese aphasia test(CCAT).

Analysis 
Statistical analysis was conducted via Statistical Product and Service Solution (SPSS) (version 21.0). Continuous variables such as length of stays in PAC were indicated as mean and standard deviation. Bivariate correlations, which were utilized for analyzing improvements of functional ability and the length of stay, were performed under Pearson product-moment correlation coefficient. Statistical significance is regarded as $p$-value less than 0.05 .

\section{Results}

We recruited 193 stroke patients with mean age of $63.07 \pm 14.135$ and with the oldest one aged 91 while the youngest one aged 26. The average duration of hospitalization in PAC was $35.01 \pm 16.373$ days with longest stay of 84 days and shortest stay of 4 days. There are $76.7 \%(n=148)$ patient of ischemic stroke and $23.3 \%(n=45)$ of hemorrhagic stroke. $60.0 \%(n=117)$ of stroke patients were male and $39.4 \%(n=76)$ were female (Table 1$)$. Among all stroke patients, only $51.8 \%(n=100)$ received speech therapy during PAC hospitalization.

As for improvements after PAC program, mean MRS decreased $0.554 \pm 0.678$, mean $\mathrm{BI}$ increased $25.839 \pm 18.585$, mean FOIS upgraded $0.495 \pm 1.038$, mean MNA raised $1.832 \pm 3.584$, mean QoL reduced 1.922 \pm 2.167 , mean IADL grew 1.352 \pm 1.350 , mean BAL rose 15.534 \pm 11.972 , mean Gait Speed extended 4.726 \pm 23.414 , mean 6 MWT surged $91.443 \pm 99.424$, mean sensory function of FMA advanced $9.358 \pm 11.785$, mean motor function of FMA elevated 11.757 \pm 11.718 , mean MMSE $3.156 \pm 4.830$, mean occupational mobility (amount use) of MAL gained $0.679 \pm 1.471$, mean occupational mobility (quality) of MAL expanded 0.278 \pm 7.924 and mean CCAT improved 0.680 \pm 1.067 (Table 2).

The longer duration of hospitalization in PAC positively correlates to $\mathrm{BI}\left(\mathrm{p}=0.000^{\star \star \star}, \mathrm{R}=0.330\right)$, $\operatorname{BAL}(p=0.000 * \star \star, R=0.461)$, Gait Speed $(p=0.002 * \star, R=0.218)$, upper sensory function of $F M A(p=0.000 \star \star \star$, $R=0.263$ ) and upper motor function of $\operatorname{FMA}(p=0.000 * * *, R=0.276)$ in stroke patients significantly(Figure 2). However, no significant correlation between duration of hospitalization in PAC and MRS( $p=0.071$, $R=-0.130), \operatorname{FOIS}(p=0.175, R=0.099), M N A(p=0.408, R=0.060), Q o L(p=0.219, R=-0.089), \operatorname{IADL}(p=0.052$, $R=0.140), 6 M W T(p=0.208, R=0.091), \operatorname{MMSE}(p=0.066, R=0.133)$, occupational mobility (amount use) of $\operatorname{MAL}(p=0.714, R=-0.027)$, occupational mobility (quality) of $\operatorname{MAL}(p=0.859, R=-0.013)$ or $\operatorname{CCAT}(p=0.060$, $\mathrm{R}=0.189$ ) were found(Table 3).

\section{Discussion}

The PAC program revealed a more economic ${ }^{2,3}$ and better recovery strategy ${ }^{4}$ for stroke patient after acute phase. Current essay indicate benefits in ADL function, balance/coordination, walking speed, motor and sensory function of upper extremities after PAC hospitalization ${ }^{1,5}$, and in our study, we found all the 
improvements of BI, BAL, Gait Speed as well as upper sensory and motor function of FMA correlate to longer stays in PAC for stroke patients.

However, though recent investigation implied advancements in MRS, FOIS, MNA, QoL, IADL, 6MWT, MMSE, occupational mobility(amount use) of MAL, occupational mobility (quality) of MAL and CCAT 5 , our data didn't pointed out positive correlation between the duration in PAC and the above improvements. Interestingly, though not statistically significant, improvements in cognition and language, measuring in MMSE and CCAT, showed positive correlations with the duration in PAC to a certain degree, as $p$ value reached 0.066 in MMSE and 0.060 in CCAT respectively.

The average length of stay in PAC in our study is $35.01 \pm 16.373$ days, which is a little longer than previous data of 25.21 34.11 days admitted in PAC for stroke patients in sub-acute phase ${ }^{3}$. Ischemic stroke accounted for $76.7 \%(n=148)$ stroke patients and hemorrhagic type accounted for $23.3 \%(n=45)$, which is quite compatible with previous essay indicated in $80 \%$ of ischemic stroke and $20 \%$ of hemorrhagic stroke in epidemiological prevalence ${ }^{6}$.In our study, stroke patients included $60.6 \%(n=117)$ male and $39.4 \%$ $(n=76)$ female. Generally, male stroke incidence and prevalence surpassed $33 \%$ and $41 \%$ than the female $e^{7}$.

One essay has shown that speech disorder could be found in $82.37 \%$ of stroke patients ${ }^{8}$ but in our study only $51.8 \%(n=100)$ receiving speech therapy and no positive relations between longer stay in PAC and improvements in CCAT. Dysphagia, on the other hand, were verified in $23 \%$ to $50 \%$ stroke patients ${ }^{9}$ and 99\% ( $\mathrm{N}=191)$ of our stroke patients underwent swallowing training. FOIS was conducted to assess oral intake function and poor oral feeding may contribute to malnutrition in a certain way. Though FOIS and MNA do show benefits for stroke patient after PAC program ${ }^{5}$, both of them do not positively correlate to the duration of hospitalization in PAC in our study simultaneously.

Gait speed had positive correlation with the length of stay in PAC in our study while 6MWT didn't. Usual gait speed is executed with regular distance $(5$ meters of valid testing, 1 meter for acceleration and 1 meter for deceleration) and recorded the time of walking to acquire walking velocity ${ }^{10}$. On the contrary, $6 \mathrm{MWT}$ is executed with regular time of 6 minutes and recorded the distance of walking within the time ${ }^{11}$. Gait speed is viewed as the sixth vital sign to assess general function and capacity of a person ${ }^{10}$ and Gait speed was found significantly and independently associated with 6- minute-walk distance in severe chronic lung disease ${ }^{12}$. Both Gait Speed and 6MWT allow patients bring their walking devices during examination but are invalid if the patients need help from other person to accomplish the examination. Both tests mainly base on patients' ambulation by themselves, but 6MWT requires better cardiopulmonary capacity to achieve greater outcome. Thus, 6MWT may not show positive correlation to the length of stay in PAC as Gait speed does, since good cardiopulmonary capacity may not be easily established early in sub-acute phase of stroke.

ADL function is represented with MRS and $\mathrm{BI}$ in acute medical center and PAC setting. Relationship between MRS and BI in stroke patients was investigated in many essays, but there's no consensus for it. 
Poor outcomes of stroke are viewed as MRS $>3$ and $B \mid<60^{13}$. In our study, MRS doesn't reveal significant correlation with the duration of hospitalization in PAC while Barthel index does. This may be elucidated with different measuring categories in these two measurements. MRS basically focus on ambulation and ability to accomplish activity before stroke attack with $0 \sim 6$ scale. On the other hand, Barthel index mainly aims on the capability to complete various aspects in daily life with $0 \sim 100$ scale. Previous essay have also pointed out MRS are mainly for global disable assessment and doesn't account for limitation in normal activity of daily life ${ }^{14}$. That is to say, Barthel index is able to reflect small degree improvements of stroke patients and thus leading to significant correlation between its improvements and the length of stay in PAC.

Upper extremities function is evaluated by motor function of FMA and MAL(amount use/ quality) in our study. Current essay indicated more common use of FMA for post- stroke upper extremities function evaluation but international consensus of standard measurement is less established ${ }^{15}$. In our study, FMA showed significant correlation between its improvements and the length of stay in PAC but MAL didn't. It could be explained by the divergent way of examining upper extremities in the two measurements. Motor function of FMA examines joint movement, i.e. wrist extension, and coordination(finger-to-nose) ${ }^{16}$. Meanwhile, MAL focus on whether the patient could finish certain tasks by their upper extremities, i.e. use a key to open a door by self-reporting ${ }^{17}$. One essay revealed that FMA is more applicable for multiple impairment of upper extremity while MAL may be influenced by patient's recall and cognition ${ }^{18}$. It takes more coordination and enough muscle power to execute tasks in MAL and thus more rehabilitation is needed after joint movements training. In the sub-acute phase of stroke patients in PAC, the improvements in the motor function of FMA could be manifest easier than MAL and therefore significant correlation between improvements in motor function of FMA and the length of stay in PAC was noted in our study.

\section{Limitation}

There are some limitations in our study. This is a retrospective and a single center research, so the results may not virtually apply to nation-wide or even worldwide stoke patients. Subgroup analysis, such as stroke type, gender and age, should be further investigated to acquire more information. Different recorders in each PAC may derive bias and so does the training efficacy variant in each institution. Last but not least, the judging scale may not fully represent the patients' recovery and each measurement had its own restriction (i.e., 6MWT couldn't represent cardiopulmonary capacity in bed-ridden stroke patients). Therefore, more survey is needed to clarify the above questions.

\section{Conclusions And Implications}

Longer duration of hospitalization in PAC is conducive to enhancing ADL function, advancing balance and coordination, boosting walking speed and augmenting both dexterity and sensory function of upper limb in stroke patients. Physiatrists and medical team members could take this result into consideration while deciding discharge time and predicting further improvements for stroke patients in clinical setting. 


\section{Abbreviations}

Activities of daily living , ADL; Barthel index, Bl; Berg Balance Test, BAL; Concise Chinese aphasia test, CCAT; EuroQoL-5D, QoL; Fugl-Meyer Assessment, FMA; Functional oral intake scale, FOIS; Lawton-Brody instrumental activity of daily life Scale, IADL; Mini-mental state examination , MMSE; Mini nutritional assessment, MNA; Modified Rankin scale, MRS; Motor Activity Log, MAL; Post-acute care, PAC; Six-Minute Walk Test, 6MWT; Usual Gait Speed, Gait Speed

\section{Declarations}

\section{Ethics approval and consent to participate}

Not applicable

\section{Consent for publication}

Not applicable

\section{Availability of data and material}

The datasets used and analysed during the current study are available from the corresponding author on reasonable request.

\section{Competing interests}

The authors declare that they have no competing interests.

\section{Funding}

There's no funding regarding this study.

\section{Authors' contributions}

Study concept and design: Hsin-Han Cheng, Willy Chou

Acquisition of data: list names: Yu-Ju Tung 
Analysis and interpretation of data: list names: Yu-Ju Tung, Hsin-Han Cheng, Willy Chou

Drafting of the manuscript: Yu-Ju Tung

Critical revision of the manuscript for important intellectual content: Wily Chou

\section{Acknowledgements}

We thank Physical medicine and rehabilitation of Chi Mei Medical Center for supporting this work.

\section{References}

1. Peng LN, Lu WH, Liang CK, et al. Functional Outcomes, Subsequent Healthcare Utilization, and Mortality of Stroke Postacute Care Patients in Taiwan: A Nationwide Propensity Score-matched Study. Journal of the American Medical Directors Association. 2017;18(11):990.e7-.e12.

2. Chan L, Sandel ME, Jette AM, et al. Does postacute care site matter? A longitudinal study assessing functional recovery after a stroke. Arch Phys Med Rehabil. 2013;94(4):622-9.

3. Wang CY, Chen YR, Hong JP, Chan CC, Chang LC, Shi HY. Rehabilitative post-acute care for stroke patients delivered by per-diem payment system in different hospitalization paths: A Taiwan pilot study. International journal for quality in health care : journal of the International Society for Quality in Health Care. 2017;29(6):779-84.

4. Wang G, Zhang Z, Ayala C, Dunet DO, Fang J, George MG. Costs of hospitalization for stroke patients aged 18-64 years in the United States. J Stroke Cerebrovasc Dis. 2014;23(5):861-8.

5. Lai CL, Tsai MM, Luo JY, Liao WC, Hsu PS, Chen HY. Post-acute care for stroke - a retrospective cohort study in Taiwan. Patient preference and adherence. 2017;11:1309-15.

6. Ojaghihaghighi S, Vahdati SS, Mikaeilpour A, Ramouz A. Comparison of neurological clinical manifestation in patients with hemorrhagic and ischemic stroke. World journal of emergency medicine. 2017;8(1):34-8.

7. Appelros P, Stegmayr B, Terent A. Sex differences in stroke epidemiology: a systematic review. Stroke. 2009;40(4):1082-90.

8. Vidovic M, Sinanovic O, Sabaskic L, Haticic A, Brkic E. Incidence and types of speech disorders in stroke patients. Acta clinica Croatica. 2011;50(4):491-4.

9. Singh S, Hamdy S. Dysphagia in stroke patients. Postgraduate medical journal. 2006;82(968):38391.

10. Middleton A, Fritz SL, Lusardi M. Walking speed: the functional vital sign. Journal of aging and physical activity. 2015;23(2):314-22. 
11. Erratum: ATS Statement: Guidelines for the Six-Minute Walk Test. American journal of respiratory and critical care medicine. 2016;193(10):1185.

12. DePew ZS, Karpman C, Novotny PJ, Benzo RP. Correlations between gait speed, 6-minute walk distance, physical activity, and self-efficacy in patients with severe chronic lung disease. Respiratory care. 2013;58(12):2113-9.

13. Sulter G, Steen C, De Keyser J. Use of the Barthel index and modified Rankin scale in acute stroke trials. Stroke. 1999;30(8):1538-41.

14. Uyttenboogaart M, Luijckx GJ, Vroomen PC, Stewart RE, De Keyser J. Measuring disability in stroke: relationship between the modified Rankin scale and the Barthel index. Journal of neurology. 2007;254(8):1113-7.

15. Santisteban L, Térémetz M, Bleton J-P, Baron J-C, Maier MA, Lindberg PG. Upper limb outcome measures used in stroke rehabilitation studies: a systematic literature review. PloS one. 2016;11(5):e0154792.

16. Sullivan KJ, Tilson JK, Cen SY, et al. Fugl-Meyer assessment of sensorimotor function after stroke: standardized training procedure for clinical practice and clinical trials. Stroke. 2011;42(2):427-32.

17. Taub E, McCulloch K, Uswatte G, et al. Motor activity log (mal) manual. UAB Training for Cl Therapy. 2011;1:18.

18. Lang CE, Bland MD, Bailey RR, Schaefer SY, Birkenmeier RL. Assessment of upper extremity impairment, function, and activity after stroke: foundations for clinical decision making. Journal of Hand Therapy. 2013;26(2):104-15.

\section{Tables}

Table 1. Demographics of stroke patients, mean length of stay in PAC and mean age

\begin{tabular}{ll}
\hline Variables & Patients $(\mathrm{n}=196)$ \\
\hline Gender & \\
Male & $117(59.7 \%)$ \\
& $76(40.3)$ \\
Female & $63.07 \pm 14.135$ years old \\
Length of stay in PAC & $35.01 \pm 16.373$ days \\
Stroke types & \\
Hemorrhagic & $45(23.3 \%)$ \\
Ischemic & $148(76.7 \%)$ \\
\hline
\end{tabular}

Table 2. Functional ability before and after Post-acute care(PAC) training program 


\begin{tabular}{llll}
\hline Variables & Before PAC program & After PAC program & Improvements \\
\hline MRS & $3.69 \pm 0.574$ & $3.13 \pm 0.874$ & $-0.554 \pm 0.678$ \\
BI & $42.64 \pm 22.873$ & $68.48 \pm 25.441$ & $25.839 \pm 18.585$ \\
FOIS & $6.03 \pm 1.532$ & $6.53 \pm 1.022$ & $0.495 \pm 1.038$ \\
MNA & $19.65 \pm 5.308$ & $21.48 \pm 4.885$ & $1.832 \pm 3.584$ \\
QoL & $10.47 \pm 2.255$ & $8.55 \pm 2.250$ & $-1.922 \pm 2.167$ \\
IADL & $1.59 \pm 1.498$ & $2.94 \pm 1.863$ & $1.352 \pm 1.350$ \\
BAL & $20.26 \pm 16.659$ & $35.80 \pm 17.317$ & $15.534 \pm 11.972$ \\
Gait Speed & $7.28 \pm 17.924$ & $12.00 \pm 16.921$ & $4.726 \pm 23.414$ \\
6MWT & $55.30 \pm 106.361$ & $146.74 \pm 153.328$ & $91.443 \pm 99.424$ \\
FMA sensory & $28.08 \pm 15.515$ & $37.44 \pm 12.616$ & $9.358 \pm 11.785$ \\
FMA motor & $27.56 \pm 22.293$ & $39.32 \pm 22.714$ & $11.757 \pm 11.718$ \\
MMSE & $18.78 \pm 8.669$ & $21.92 \pm 7.704$ & $3.156 \pm 4.830$ \\
MAL(amount use) & $0.87 \pm 1.255$ & $1.55 \pm 1.926$ & $0.679 \pm 1.471$ \\
MAL(quality) & $1.51 \pm 7.499$ & $1.79 \pm 3.173$ & $0.278 \pm 7.924$ \\
CCAT & $9.84 \pm 2.387$ & $10.42 \pm 2.028$ & $0.680 \pm 1.067$ \\
\hline
\end{tabular}

Abbreviation: MRS, modified Rankin scale; BI, Barthel index; FOIS, functional oral intake scale; MNA, mini nutritional assessment; QoL, EuroQoL-5D; IADL, Lawton-Brody IADL Scale; BAL, Berg Balance Test; Gait Speed, Usual Gait Speed; 6MWT, Six-Minute Walk Test; FMA, Fugl-Meyer Assessment; MMSE, mini-mental state examination; MAL, Motor Activity Log; CCAT, concise Chinese aphasia test

Table 3. Improvements of the functional domains after PAC program and the correlations with the length of stay in PAC

\begin{tabular}{|c|c|c|}
\hline & $\mathrm{p}$ & $\bar{R}$ \\
\hline$\Delta \mathrm{MRS}$ & 0.071 & -0.130 \\
\hline$\Delta \mathrm{BI}$ & $0.000 * * *$ & 0.330 \\
\hline$\Delta$ FOIS & 0.175 & 0.099 \\
\hline$\triangle \mathrm{MNA}$ & 0.408 & 0.060 \\
\hline$\Delta \mathrm{QoL}$ & 0.219 & -0.089 \\
\hline$\triangle \mathrm{IADL}$ & 0.052 & 0.140 \\
\hline$\triangle \mathrm{BAL}$ & $0.000 * * *$ & 0.461 \\
\hline$\Delta$ Gait Speed & $0.002^{* *}$ & 0.218 \\
\hline$\Delta 6 \mathrm{MWT}$ & 0.208 & 0.091 \\
\hline$\Delta$ FMA sensory & $0.000 * * *$ & 0.263 \\
\hline$\Delta \mathrm{FMA}$ motor & $0.000 * * *$ & 0.276 \\
\hline$\triangle \mathrm{MMSE}$ & 0.066 & 0.133 \\
\hline$\Delta \mathrm{MAL}$ (amount use) & 0.714 & -0.027 \\
\hline$\triangle \mathrm{MAL}$ (quality) & 0.859 & -0.013 \\
\hline$\triangle \mathrm{CCAT}$ & 0.060 & 0.189 \\
\hline
\end{tabular}

Abbreviation: SD, standard deviation; MRS, modified Rankin scale; BI, Barthel index; FOIS, functional oral intake scale; MNA, mini nutritional assessment; QoL, EuroQoL-5D; IADL, Lawton-Brody IADL Scale; BAL, Berg Balance 


\section{Figures}

\section{5 stroke patients eligible for Post-Acute Care(PAC) admission}

\section{8 patients ended PAC admission earlier due to stroke or non-stroke disease progression}

\section{6 stroke patients}

3 stroke patients refused to PAC admission;

6 stroke patients had recurrent stroke during acute phase

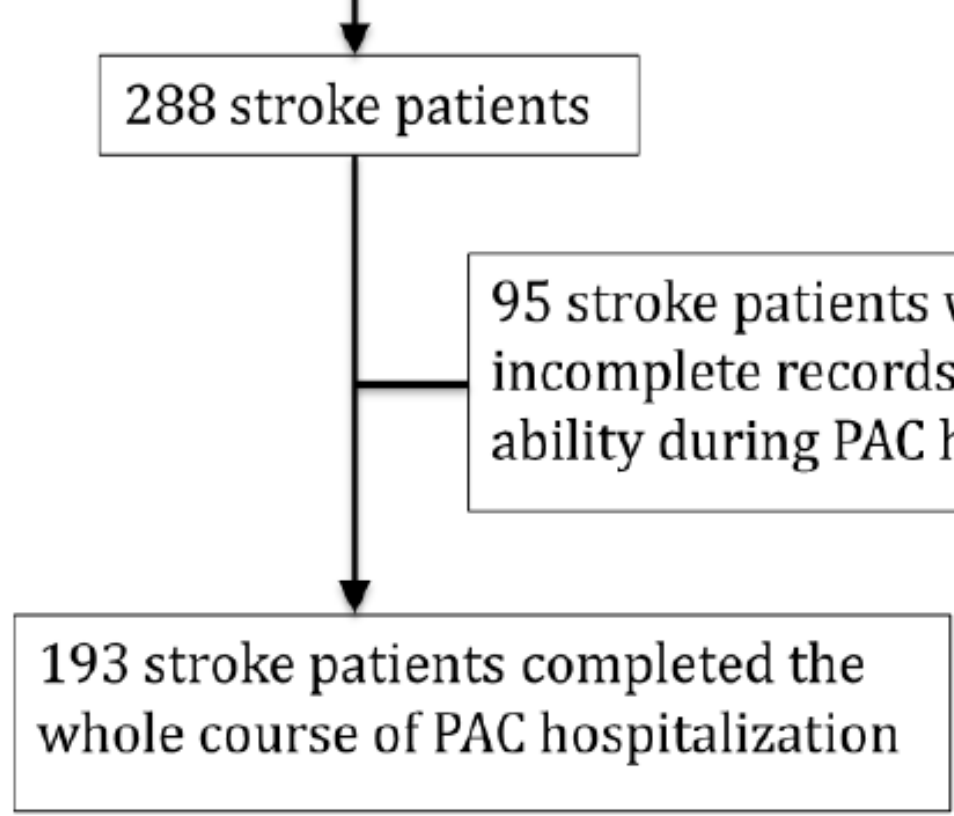

Figure 1 
Enrollment diagram of the study.
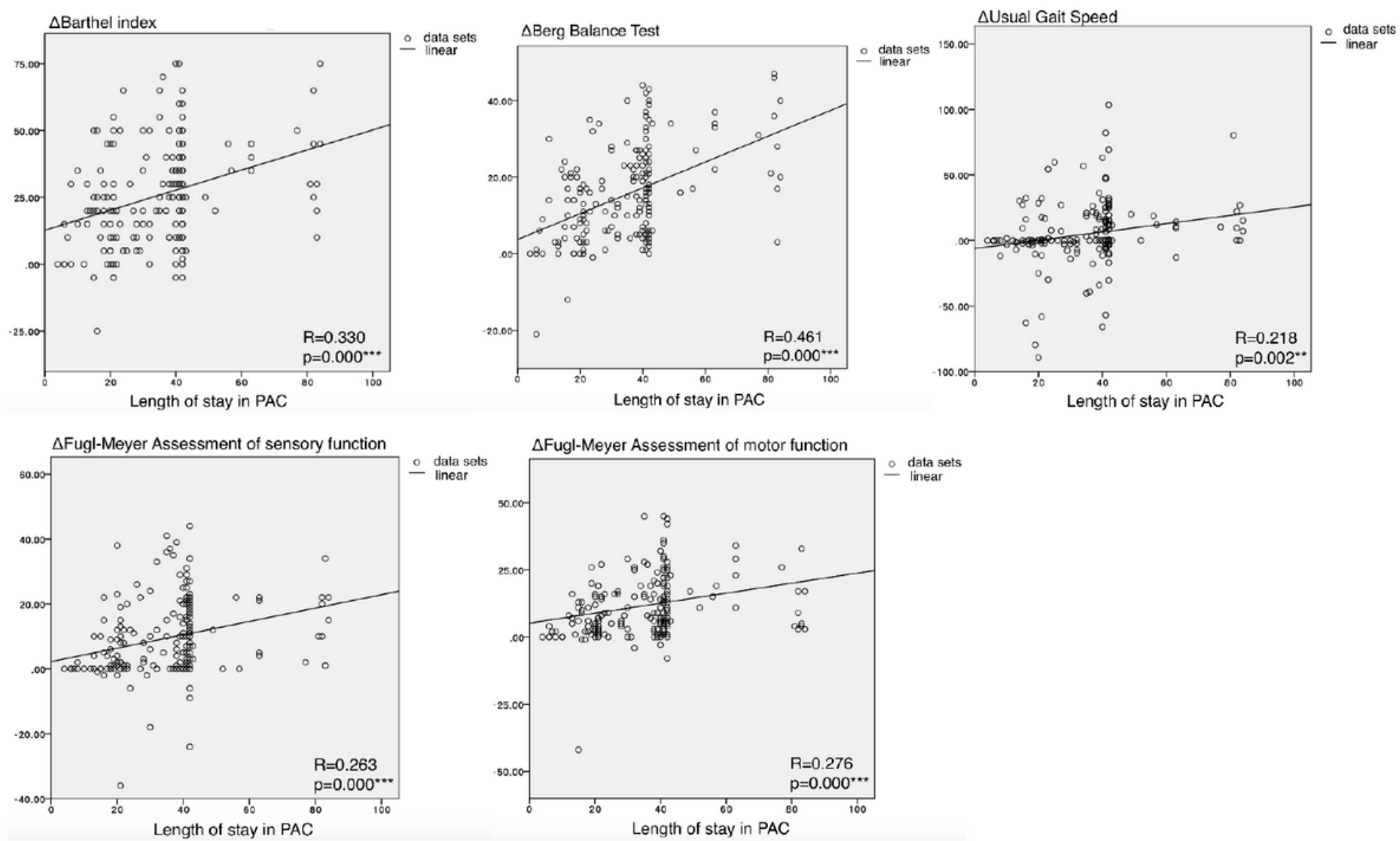

Figure 2

Correlation between $\Delta$ Barthel index, $\Delta$ Berg Balance test, $\Delta$ Usual Gait Speed, $\Delta$ Fugl-Meyer Assessment of sensory and motor function and the length of stay in PAC

\section{Supplementary Files}

This is a list of supplementary files associated with this preprint. Click to download.

- STROBEChecklistPageBrackets.pdf 